\title{
Comparative Study between the Algorithm using Frequency Domain and the Algorithm using Linear Programming
}

\author{
Mona M. Abd El-kareem ${ }^{1 *}$ and Ibrahiem M. M. El-Emary² \\ 'Management Information System and Computer Science Dep., Future Academy, Cairo, Egypt; \\ Mona.abdelkareem@fa-hists.edu.eg \\ 2Information Technology Deanship, King Abdulaziz University Jeddah, Kingdom of Saudi Arabia; \\ omary57@hotmail.com
}

\begin{abstract}
Background/Objectives: Comparative study is a research methodology in the social sciences that aims to make comparisons across different countries or cultures. A major problem in comparative research is that the data sets in different countries may not use the same categories or define categories differently. Methods: A systematic design for comparing the performance of Filtering Algorithms methods is used to for maximum measurement of frequencies in the $x$-direction, $y$-direction which are connected to the minimum grid distance in the $x$ and $y$ direction changing the minimum grid distance used in the $x$ and $y$ direction. Findings: These frequencies are the ones used in the deciding the lowest and highest frequencies chosen in the $\mathrm{x}$ and $\mathrm{y}$ directions respectively. Applications: To illustrate the benefit of using different filters, we give results of filtering applied to certain image.
\end{abstract}

Keywords: Filters, Frequency Domain, Linear Programming, Z-transform

\section{Introduction}

Time-domain and frequency domain representation methods offer alternative insights into a system and depending on the application it may be more convenient to use one method in preference to the other. Time domain system analysis methods are based on differential equations which describe the system output as a weighted combination of the differentials (i.e. the rates of change) of the system input and output signals ${ }^{1}$. The description of a system in the frequency domain can reveal valuable insight into the system behavior and stability ${ }^{2}$.

\section{A General Z-transform Formula}

We have seen that for a sequence $x[n]$ having support interval $0 \leq n \leq N$ the $\mathrm{z}$-transform is ${ }^{3}$.

$$
X(z)=\sum_{n=0}^{N} x[n] z^{-n}
$$

This definition extends for doubly infinite sequences having support interval $-\infty \leq n \leq \infty$ to

$$
X(z)=\sum_{-\infty}^{\infty} x[n] z^{-n}
$$

\subsection{Relationship Between the z-Domain and} the Frequency Domain

$$
\begin{array}{lr}
\hat{\omega} \text { - Domain. } & z \text { - Domain } \\
H\left(e^{j \hat{\omega}}\right)=\sum_{k=0}^{M} b_{k} e^{-j \hat{\omega} k} \text { Versus } & H(z)=\sum_{k=0}^{M} b_{k} z^{-k}
\end{array}
$$


comparing the above we see that the connection is setting $z=e^{j \hat{\omega}}$ in $H(z)$

$$
H\left(e^{j \hat{\omega}}\right)=\left.H(z)\right|_{z=e^{j \hat{\omega}}}
$$

When we make the substitution $z=e^{j \hat{\omega}}$ in $H(z)$ we know that we are evaluating the $z$-transform on the unit circle and thus obtain the frequency response. If we plot say $|H(z)|$ over the entire z-plane we can visualize how cutting out the response on just the unit circle, gives us the frequency response magnitude.

\section{Nulling Filters}

Figure 1 shows the special case of zeros on the unit circle allows a filter to null/block/annihilate complex sinusoids that enter the filter at frequencies corresponding to the angles the zeros make with respect to the real axis in the $z$-plane (Figure 2).

The nulling property extends to real sinusoids since they are composed of two complex sinusoids at $\pm \hat{\omega}_{0}$ and zeros not on the real axis will always occur in conjugate pairs if the filter coefficients are real. This nulling/annihilating property is useful in rejecting unwanted jamming and interference signals in communications and radar applications ${ }^{4}$.

\subsection{Properties of Linear-Phase Filters}

A class of FIR (Finite Impulse Response) filters having symmetrical coefficients, i.e., $b_{k}=b_{M-k}$ for $k=0,1, \ldots, M$ has the property of linear Phase.

\subsection{The Linear Phase Condition}

For a filter with symmetrical coefficients we can show that $H\left(e^{j \hat{\omega}}\right)$ is of the form $H\left(e^{j \hat{\omega}}\right)=R\left(e^{j \hat{\omega}}\right) e^{-j \omega M / 2}$

Where $R\left(e^{j \hat{\omega}}\right)$ is a real function.

The fact that $R\left(e^{j \hat{\omega}}\right)$ is real means that the phase of $H\left(e^{j \hat{\omega}}\right)$ is a linear function of frequency plus the pos- sibility of $\pm \pi$ phase jumps whenever $R\left(e^{j \hat{\omega}}\right)$ passes through zero.

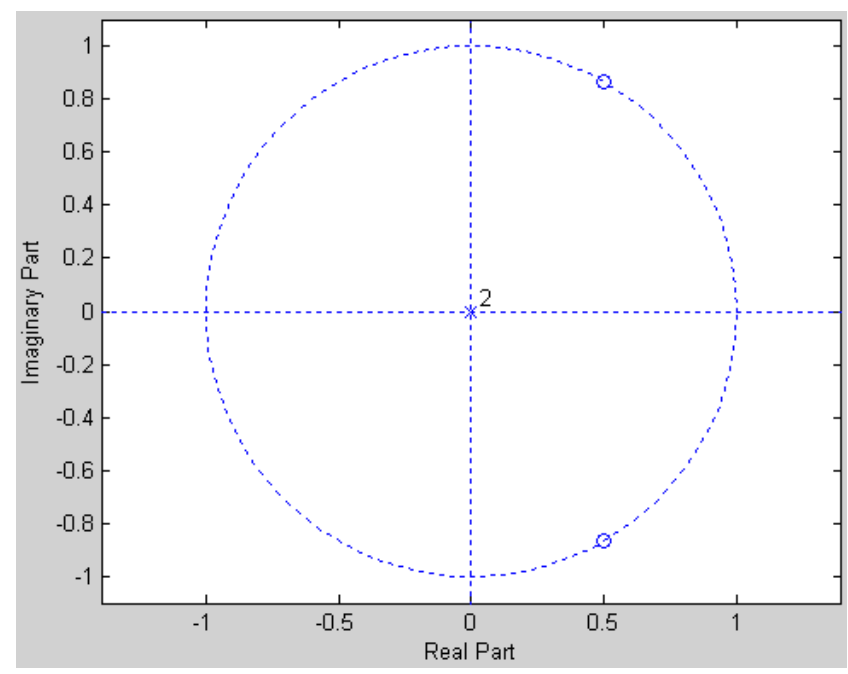

Figure 1. Nulling filter.

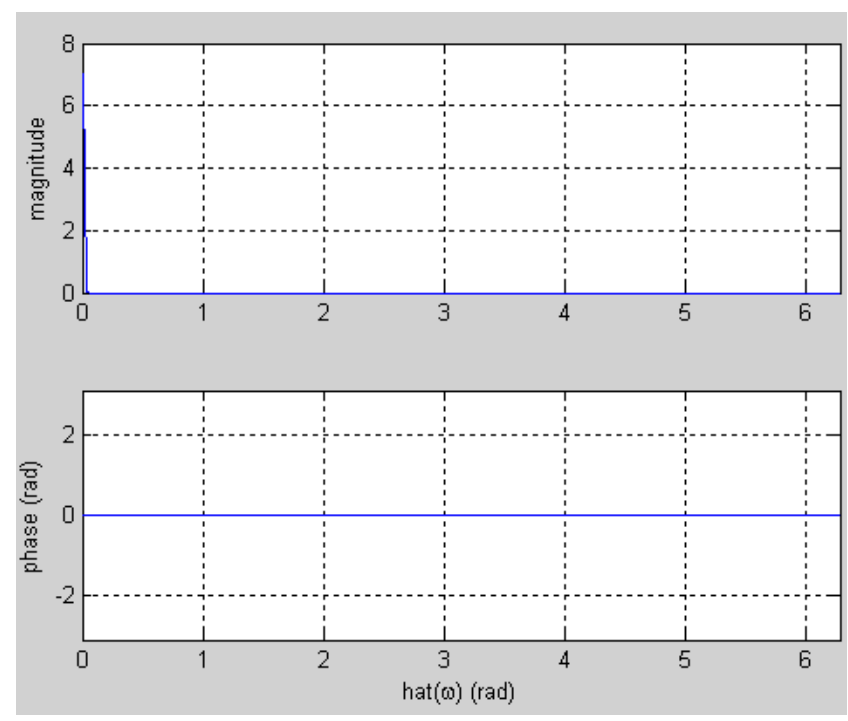

Figure 2. Sinusoidal response of FIR system.

\subsection{Linear Programming (LP)}

Many management decisions involve trying to make the most effective use of organization resources. These resources include Machinery, Labors, Money, Time, Warehouse space or Raw materials to produce goods (machinery, furniture, food or cooking) or service (schedules for machinery and production advertising policies or investment decision $)^{5}$. 
Linear Programming (LP) is a widely used mathematical techniques designed to help managers in planning and decision making relative to resource allocations ${ }^{6}$.

\subsection{Properties of Linear Programming Model}

All linear programming models have four basic properties in common. They are:

- All LP models seek to maximize or minimize some quantity, usually profit or costs.

- All LP models have constraints or limitations that limit the degree to which the objective can be purse. E.g. Deciding how many units of product in a product line to be produced is restricted to the manpower and machinery available.

- There must be alternative course of action to choose from e.g. if there are 4 different product, management may decide (using LP) how to allocate limited resources among them.

- Objectives and constraints in LP model must be expressed in linear equations and inequalities.

\subsection{Assumption of Linear Programming}

\subsubsection{Certainty}

We assume that a number in the objective and constraints are known with certainty and does not change during the study period.

\subsubsection{Proportionality}

We are sure that proportionality exists in the objective and the constraints. This means that, if production of one unit of product uses two of a particular scare resource; then making five units of that product uses ten resources.

\subsubsection{Additivity}

This means that the total of all activities equals the sum of each individual activity.

\section{Divisibility}

This means that solution may take fractional values and need not be in whole numbers (integers). If a fraction of a product cannot be produced, integer programming problem exist.

\subsection{Formulation of Linear Programming Model}

Linear programming problems are optimization problem which are stated verbally or in words. The following steps can be used to formulate the model of any optimization problem ${ }^{?}$.

\section{Step 1}

Choose variables and notations that will be used to form the objective and constraints functions.

\section{Step 2}

Identify the objective function to either maximize or minimize e.g. maximizes profit or minimizes cost.

\section{Step 3}

Develop mathematical relationships to describe objective and constraints.

\section{The Proposed Methodology of Filtering Algorithms}

We describe a systematic design for comparing the performance of Filtering Algorithms methods. We show that the variable selection process is integral in comparing methods to ensure minimal bias and optimal performance. We show that, for sufficiently large samples, this bias is minimized so that methods can be compared; previous sample size recommendations are insufficient.

\subsection{Filtering Algorithms using Linear} Programming

\begin{tabular}{|l|l|}
\hline Input & Noisy image \\
\hline Output & Filtered image \\
\hline $\begin{array}{l}\text { Step 1 } \\
\text { Step 2 } \\
\text { Step 3 }\end{array}$ & $\begin{array}{l}\text { Read image } f(x, y) \text { with .dat extension. } \\
\text { Determined } d_{1} \text { and } d_{2} \text { using the TORA } \\
\text { software. } \\
\text { Computing frequency domain } \omega=\frac{2 \pi C}{d_{1,2}}\end{array}$ \\
Step 4 & $\begin{array}{l}\text { Using the Z-transform to convert from time } \\
\text { domain to the frequency domain } z=e^{i \omega}\end{array}$ \\
Step 5 & $\begin{array}{l}\text { For i=1: image size } \\
\text { J }=1: \text { image size } \\
\text { Apply our proposed methodology } \\
\text { end }\end{array}$ \\
\hline
\end{tabular}


In this algorithm we read image with data extension after that we need to determined $d_{1}$ and $d_{2}$ using the TORA software then, we take the grid distance we obtained from the software to computing frequency domain and using the Z-transform to convert from time domain to the frequency domain. Finally apply our proposed methodology.

\subsection{Filtering in the Frequency Domain}

In this section we shall explore some filtering by this method.

\subsection{Filtering Algorithms using Frequency Domain}

\begin{tabular}{|l|l|}
\hline Input & Noisy image \\
\hline Output & Filtered image \\
\hline Step 1 & Read image $f(x, y)$. \\
Step 2 & Compute Fourier transform $F(u, v)$ of the image. \\
Step 3 & For i=1: image size \\
& J = 1: image size \\
& Compute a filter function $H(u, v)$ \\
Step 4 & Multply $\mathrm{F}(\mathrm{u}, \mathrm{v})$ by a filter function $H(u, v)$ \\
& $G(u, v)=F(u, v)^{\star} H(u, v)$. \\
& Compute the inverse Fourier transform \\
& Filtered image $=[G(u, v)]^{-1}$. \\
\hline
\end{tabular}

Given the filter $\mathrm{H}(\mathrm{u}, \mathrm{v})$ (filter transfer function) in the frequency domain, the Fourier transform of the output image (filtered image) is given by:

$$
G(u, v)=H(u, v) F(u, v)
$$

\section{Results and Discussion}

\subsection{Acceptance Criterion for the Filtered Images}

We use root mean square error method to know how much the filtered image is close to the original image.

We used different tolerance values make the filtered image as closed as we can to the original image and thus could control the closeness of the filtered image from the original one Figure 3.

- Choosing random tolerance to comparison between two algorithms on the range (0:5).
- Choosing random tolerance to comparison between two algorithms on the range (0:10).
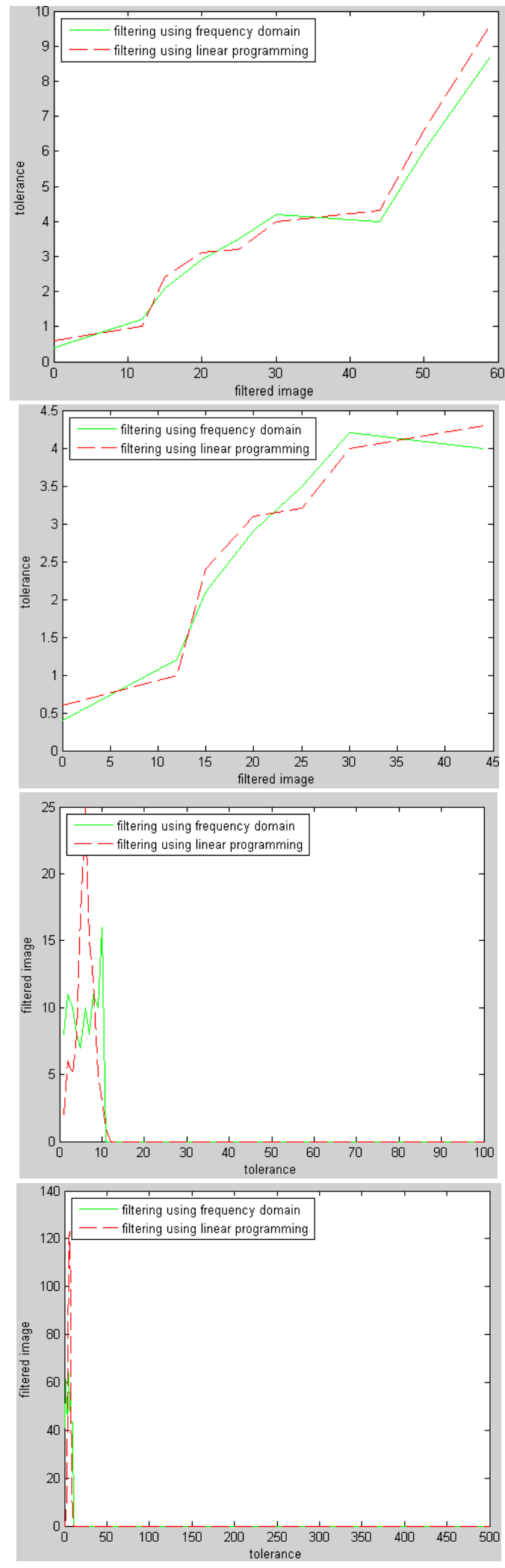


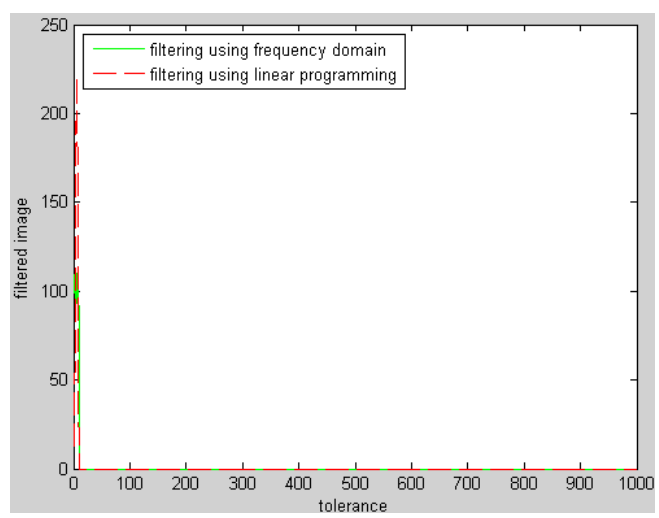

Figure 3. Relationship between tolerance and filtered image.

When we choose a random tolerance between (0:5) the results we obtained very clear, but if we discussed the difference between the algorithms we will notice that our algorithms give the best filter of the digital image in a fast way and The results are satisfactory. When we applied traditional algorithms some of them did not give satisfactory results and the most important problem is the time taken to choose the cutoffs which can be used in filters, also which filter can be used to remove the noise from the image?

This question is answered by using the TORA linear programming software that gives the proper values for $d_{1}$ and $d_{2}$ for any filter. Having obtained the values of $d_{1}$ and $d_{2}$ by using the TORA linear programming software, we apply our digital filters directly to get the best filtered values of our image.

\subsection{Limitation of the Algorithms}

Here we will discuss the results we obtained after increasing the range of the random tolerance and their impact on the images.

- Choosing random tolerance to comparison between two algorithms on the range (0:100).

There is a clear impact on the results, where we see that the results in some of the dots appeared, but only after it has become all the values given the results of a fixed. This produces when we expand or increase the size range which we choose.

- Choosing random tolerance to comparison between two algorithms on the range (0:500).

We notice from the results obtained that the program is given only the first ten values, then the values are all equal to zero until the end of the range.
- Choosing random tolerance to comparison between two algorithms on the range (0:1000).

\section{Conclusion and Future Work}

In this study we showed output result with the help of chart that the greater range 10 results have become 'close to zero and the appearance of the image contrast would not be good. This confirms that the lower of $d_{1}$ and $d_{2}$, which reflects the cutoffs whenever the best image contrast.

As a future work we would like to determine the defected region or patterns in an image according to some specified criterion

\section{Acknowledgement}

This paper has been presented and discussed during the International Conference on Communication, Management and Information Technology - ICCMIT 2019, Vienna, Austria.

\section{References}

1. Fazarinc Z. Z-transform and its application to development of scientific simulation algorithms. Computer Applications in Engineering Education. 2013; 21(1):75-88. https://doi. org/10.1002/cae.20452

2. Digital Signal Processing. 2019. https://en.wikipedia.org/ wiki/Digital_signal_processing

3. Pan Z, Cheng Y, Liu J. Stress analysis of a finite plate with a rectangular hole subjected to uniaxial tension using modified stress functions. International Journal of Mechanical Sciences. 2013; 75:880-90. https://doi.org/10.1016/j.ijmecsci.2013.06.014

4. Digital Filters and Z Transforms. 1999. https://faraday. physics.utoronto.ca/GeneralInterest/Harrison/TimeSeries/ DigFilters.pdf

5. Operations Research. 2019. https://en.wikipedia.org/wiki/ Operations_research

6. Xu W, Huang P, Wang R. TOPS - Mode raw data processing using chirp scaling algorithm. IEEE Journal of Selected Topics in Applied Earth Observations and Remote Sensing. 2014; 7:235-46. https://doi.org/10.1109/ JSTARS.2013.2260134

7. Fazarinc Z. Z-transform and its application to development of scientific simulation algorithms. Computer Applications in Engineering Education. 2013; 21(1):75-88. https://doi.org/10.1002/cae.20452 